\title{
Fator de impacto: Importância e influência no meio editorial, acadêmico e científico
}

Journal Impact factor: its editorial, academic and scientific importance

Milton A. Ruiz

Oswaldo T. Greco ${ }^{2}$

Domingo M. Braile

\begin{abstract}
Neste relato os autores apresentam informações sobre os instrumentos bibliométricos e a sua importância na aferição da qualidade dos periódicos científicos, pesquisadores e docentes. Apresentam em particular a história e a implantação do fator de impacto do Institute for Scientific Information, existente desde 1955. São apresentados e discutidos as críticas referentes à inadequação do fator de impacto para avaliação da produção cientifica, uso indevido e estratégias editoriais de manipulação deste indice bibliométrico. É apresentada a nova classificação Capes para os periódicos, baseada em vários critérios e no Fator de Impacto, em especial, e a sua influência na vida acadêmica e cientifica nacional. Conclui que, apesar de todos os óbices e discussões, o Fator de Impacto do Institute for Scientific Information ainda é uma ferramenta útil e, isoladamente, a única existente para avaliar os periódicos cientificos e a produtividade intelectual. Rev. Bras. Hematol. Hemoter.
\end{abstract}

Palavras-chave: Fator de Impacto; índices de citação; instrumentos bibliométricos.

\section{Introdução}

No editorial de fevereiro de 2008 do Clinics, o editor científico informava, em alusão à citação de capa, que a revista, no seu quarto ano de existência, estava incluída e indexada no Journal Citation Reports (JCR) do Institute for Scientific Informations (ISI). ${ }^{1}$ Ressaltava o editor que a inclusão da revista na base de dados do ISI era a última indexação que faltava ao Clinics, e assim o Fator de Impacto (FI) da revista passaria a ser medido a partir de 2009. Informava também que o FI extraoficial da revista era de 0,8 , enfatizando que tal resultado colocaria a Clinics em boa posição frente às 23 revistas brasileiras da época, indexadas naquela base de dados. Citava também o fato da existência de poucas revistas científicas nacionais com valor superior a 1,0.

Em recente comunicado de 17/09/2009 divulgado na internet pelos editores da Nature a todos editores de revistas e endereços do seu mailing list, informa que a revista foi considerada a primeira do ranking da área de periódicos multidisciplinares de ciência. Informa no corpo da mensagem que o seu novo fator de impacto referente ao ano de 2008 é de 31,434 , estando na sequência a Cell, com 31,253, e a Science, $\operatorname{com} 28,103$.

A par desta informação, os editores citaram que, além do FI, existem outros dados bibliométricos que também atestam o sucesso da Nature e concluem a sua mensagem oferecendo um desconto de $30 \%$ para novos subscritores como motivação de celebração da confirmação do periódico científico ser o mais bem classificado entre todas as revistas da área (vide apêndice).

Corroborando estas informações, editores científicos de revistas brasileiras ${ }^{2}$ têm apontado como objetivo primordial a entrada de seus periódicos na base de dados do ISI e do Medline, o que atesta não só uma tendência de aprimoramento, mas de uma evidente internacionalização dos periódicos científicos brasileiros.

${ }^{1}$ Editor da Revista Brasileira de Hematologia e Hemoterapia.

${ }^{2}$ Médico. Editor da Revista Latino Americana de Arritmia e Marcapasso (Relampa)

${ }^{3}$ Médico. Editor da Revista Brasileira de Cirurgia Cardiovascular.

Correspondência: Milton Artur Ruiz

Avenida Dr. Enéas Carvalho de Aguiar $n^{\circ} 44$ - Cerqueira César

05403-000- São Paulo-SP - Brasil

E-mail:milruiz@yahoo.com.br

Doi: 
Com este preâmbulo, que pode ser mensurado pelas informações acima citadas, é que justificamos a elaboração deste artigo, de caráter educativo, com o objetivo de disseminar informações sobre os instrumentos bibliométricos mais importantes utilizados, em especial o FI, pontuando a sua história, a relevância e as restrições que existem a este indicador assim como a sua influência no meio acadêmico, editorial e científico.

Este texto contou com a participação de três editores científicos de revistas brasileiras que consideram o momento como relevante para este tipo de divulgação que exige uma profunda reflexão sobre tema no meio editorial acadêmico e científico nacional.

\section{Instrumentos bibliométricos}

Bibliometria é o estudo dos aspectos quantitativos da produção intelectual, disseminação e o uso da informação registrada. ${ }^{3}$

Cientometria é a ciência que se destina a analisar de forma abrangente a produção científica e tecnológica utilizando diversos indicadores e instrumentos bibliométricos matemáticos com o objetivo de mensurar e compreender a dimensão deste universo. É o estudo dos aspectos quantitativos da ciência como uma disciplina ou atividade econômica. A cientometria é considerada um segmento da sociologia da ciência, sendo aplicada no desenvolvimento de políticas científicas. Envolve estudos quantitativos das atividades científicas, incluindo as publicações, e por isto sobrepõemse à bibliometria. ${ }^{3} \mathrm{O}$ Fator de Impacto (FI) dos periódicos científicos é um dos instrumentos bibliométricos existentes e tem como objetivo precípuo aferir a produção científica dos autores, a qualidade das publicações e presuntivamente classificar os periódicos científicos inseridos no Journal Citations Reports do ISI. ${ }^{4}$

O conhecimento científico e o início das formas de codificação e organização padronizada de documentações para consulta, e uso objetivo das informações, remonta a 1873, com o advento do Shepards Citations, da companhia do mesmo nome, de Colorado Springs, nos Estados Unidos da América. O periódico foi idealizado para coletar documentações jurídicas dos processos norte-americanos e se baseava em precedentes que, paulatinamente, se assomavam aos processos que iam ocorrendo nos 48 estados americanos daquela época. Além desta publicação, a Shepards Citations Incorporation editava publicações especiais, e, dentre elas, uma denominada Journal of the Patient Office Society. ${ }^{5}$

Em 1927, Gross \& Gross sugeriram que fosse realizada a contagem do número das citações de cada artigo ou trabalho, com o objetivo de classificar e determinar a importância dos periódicos científicos da época. ${ }^{6}$

Vanevar Bush, em 1945, foi o primeiro autor a propor o método da coleta, sistematização e recuperação de dados. ${ }^{7}$ Na sequência, teve início um projeto do governo dos Esta- dos Unidos, denominado Welsh Project, que redundou na organização da National Library of Medicine do jeito como a conhecemos atualmente e contou com a participação de Eugene Garfield. ${ }^{5}$ Este autor, com esta atividade e o conhecimento das experiências anteriores, foi o idealizador do FI em 1955 e o fundador do ISI. ${ }^{8}$ Logo em seguida foi publicado o Genetics Citation Index, publicação esta que propiciou o aparecimento, em 1961, do Science Citation Index e dele derivou o Journal Impact Factor (JIF), ou simplesmente FI, com o objetivo de criar um instrumento de seleção e avaliação dos periódicos científicos como já citado anteriormente. ${ }^{9}$

O cálculo do FI de uma revista para um determinado ano X (Tabela 1) é obtido com a divisão do número de citações dos artigos de uma revista em todas as revistas inseridas na base de dados do ISI, dividido pelo que foi publicado por esta revista nos dois anos anteriores. Assim, para exemplificarmos e utilizando o exemplo da Clinics, o primeiro fator de impacto da revista a ser publicado será em 2011 e referente a 2010, pois os anos que passarão a ser contabilizados para o cálculo serão os anos de 2009 e 2010.

Tabela 1. Fórmula de cálculo do Fator de Impacto anual

$\mathrm{FI}$ do ano $\mathrm{X}=\mathrm{N}^{\circ}$ de citações do periódico obtidas nos dois anos anteriores

$\mathrm{N}^{\circ}$ artigos (substanciais ${ }^{*}$ ) publicados nos dois anos anteriores

Existem mais de 30 índices de aferições existentes além do FI e, dentre todos, segue sendo o mais utilizado o Índice de Imediatez (Ii), além dos índices de obsolescência, como a vida média dos artigos, sendo todos reportados no Journal Citation Reports (JCR), que está atualmente no portfólio da Thomson Reuters Company.

O Índice de Imediatez (Ii), também conhecido como Índice de Imediaticidade ou de Fator de Impacto Imediato, refere-se à frequência com que os artigos de uma revista são citados pelas outras revistas no mesmo ano de sua publicação. O cálculo deste índice é simples e obtido com a divisão do total de citações da referida revista nos periódicos abrangidos pelo ISI, divididos pelo total de artigos publicados pela revista no próprio ano. Este índice é o primeiro a ser disponibilizado pelo ISI e é publicado logo no primeiro ano de indexação de uma revista em sua base de dados.

$\mathrm{O}$ índice h é um outro índice bibliométrico que tem o objetivo de quantificar a produtividade e o impacto de cientistas baseado nos seus artigos mais citados.

O objetivo do índice é o de estimar a produtividade e o impacto de um ou mesmo de um grupo de cientistas. O índice, proposto em 2005 por Jorge E. Hirsch, visava ser uma ferramenta para determinar a qualidade dos trabalhos de físicos teóricos. Apesar de ter que suplantar outros índices e determinações tradicionais, como a enumeração do número de artigos publicados por um autor, o número total de citações obtidas pelos artigos deste autor e avaliação do impac- 
to das revistas onde autor publica seus trabalhos, este índice paulatinamente vem ganhando adeptos.

$\mathrm{O}$ índice h calcula-se pela observação do número de artigos com citações maiores ou iguais a um determinado número. Exemplificando, um pesquisador, para ter um índice $\mathrm{h}$ de 5 , terá de possuir em seu currículo pelo menos cinco artigos que tenham recebido cinco ou mais citações. Este índice tem como atributo principal a avaliação global da produtividade do autor independente do local de sua publicação. Assim, um autor que tenha publicado somente dois artigos, sendo um, p.ex., digamos, na Nature, onde obteve deste artigo um total de 238 citações, e um outro em uma revista obscura e desconhecida, na qual obteve somente duas citações; com este desempenho, o índice h do autor seria de 2 por não possuir mais de dois artigos com mais de duas citações. Esta é a principal crítica ao índice h, pois o desempenho do autor com o aumento de sua produção dependendo do local em que o mesmo publique poderá cair, sendo pertinente publicar para divulgar e ser citado para não ser esquecido, e este índice serve de exemplo para mensuração global de um autor ou de um grupo de pesquisadores. Publique para existir e seja citado para não desaparecer ou ser esquecido. ${ }^{10}$

\section{O Fator de Impacto e a sua relevância}

Assim como exemplificamos anteriormente, o FI de um determinado ano tem, em sua composição, o número de citações de artigos ou de comunicados científicos de um determinado periódico no universo de todos periódicos nos dois anos anteriores como numerador, e, como denominador, os artigos publicados pelo periódico neste mesmo período. ${ }^{9} \mathrm{~A}$ sua importância para o mercado editorial pode ser medido pelo histórico do ocorrido com o Journal of the American Medical Association (JAMA) no início da década de 80. Considerado um periódico científico de grande prestígio na década de 60, George Lundberg, então novo editor, observou uma queda da popularidade da revista pela redução de citações dos artigos da revista e consequente redução do FI. Com esta observação, o editor propôs mudanças na política editorial na revista, que abrangiam o conteúdo, a forma de submissão e de aceitação de artigos, além de criar novas seções para a revista. Passou principalmente a dedicar um grande cuidado em relação aos artigos submetidos, principalmente aos passíveis de serem citados ou com potencial de entrarem na composição do cálculo do FI. O editor também se tornou mais agressivo no contato com os autores considerados formadores de opinião, com o objetivo precípuo de atrair artigos, e de ter os artigos publicados no JAMA por estes autores citados em outros periódicos indexados na base de dados do ISI. Esta iniciativa, que se tornou clássica, é ainda o paradigma da atuação dos editores científicos no mercado editorial. Outro aspecto a ser observado é o frisson do meio do ano em que os editores de revistas e publishers do mundo inteiro esperam ansiosamente pela divulgação do FI na Philadelphia - USA, pelo ISI (apêndice).

O FI, como uma simples medida de qualidade das revistas, ao longo do tempo passou a ser utilizado como uma ferramenta acadêmica de avaliação de produtividade e também para obtenção de fundos. Assim, governos e agências de fomento de diversos países passaram a utilizar o FI como instrumento de decisão para alocação de recursos aos pesquisadores. Em suma, tornou-se um guia de política científica. Esta tendência foi observada durante vários anos no Reino Unido, onde o índice foi utilizado intensamente na pesquisa e na educação. ${ }^{11,12}$ Este fato também está ocorrendo no Brasil, onde o FI gradualmente evoluiu para representar tanto o fator de impacto do periódico como também de produtividade do próprio autor. $\mathrm{O}$ índice, hoje, é utilizado para avaliar os autores e também como ferramenta acadêmica e de classificação dos pesquisadores e docentes, que passaram a ser classificados segundo suas publicações nos periódicos com alto, baixo ou sem impacto de suas publicações.

Como exemplo do citado, a Coordenação de Aperfeiçoamento de Pessoal de nível superior (Capes) publicou, no ano passado, uma nova forma de classificação e de estratificação dos periódicos científicos nacionais e internacionais. A classificação para todas as áreas temáticas é composta de 8 níveis, que vão de $\mathrm{A} 1$ e $\mathrm{A} 2$, considerados os mais altos, havendo poucas revistas com esta classificação, e de B1 a B5 até o nível C, considerado o mais baixo. ${ }^{13}$

Para esta estratificação foram utilizados os parâmetros formais de avaliação do periódico científico, como formato, existência de número de registro no International Standard Serial Number (ISSN), periodicidade, conteúdo científico representativo, corpo editorial com qualificação, e se os artigos publicados no periódico são revisados por pares além do seguimento das normas da Organização Mundial dos Editores Científicos (WAME) denominado, no passado, de grupo de Vancouver.

Foi avaliada também a inserção dos periódicos nas bases de dados da National Library of Medicine, Pub Med/ Medline, ISI e SciELO, Lilacs e outras.

Dentre os índices bibliométricos para estratificar as revistas, o mais importante dos utilizados foi o FI, o que colocou todos os periódicos nacionais da área de ciências da saúde abaixo de B2 por terem os periódicos nacionais FI abaixo de 1,5 . Os periódicos que não estão na base de dados da ISI foram considerados B4, e os que não estão na coleção SciELO, abaixo de B5.

Assim, o FI norteou a conduta do Capes e esta passou a influenciar todos os programas de pós-graduação do País, que foram reclassificados. O programa de pós-graduação de várias instituições, após esta nova classificação, passou a rever a produtividade do seu corpo docente e pontuar seus orientadores segundo a nova regra. Em vários deles foram traçadas novas metas, obrigando orientadores e orientados a publicar seus resultados em revistas que tenham as mais 
altas classificações. Existem relatos, e isto tem sido observado, de obrigatoriedade para conclusão, em algumas instituições de doutorado, de publicações em revistas com classificação B1 ou superior, o que equivale à publicação em revistas com FI superior a 3,0.

Como observamos, o FI passou a permear, dirigir e interferir na produção acadêmica e científica do País.

\section{Discussão}

O FI, idealizado inicialmente como um sistema bibliográfico para as literaturas de ciência com o objetivo de eliminar citações que não fossem criteriosas, fraudulentas, incompletas ou que contivessem dados obsoletos para uso escolar ou de futuros artigos, ${ }^{8}$ com o passar do tempo extrapolou esta função. Além de ser um instrumento de qualificação dos periódicos, passou também a ser o norteador de medidas educacionais, acadêmicas e de base para muitos países, como o Brasil, e, principalmente, de parâmetro de decisões de agências de fomento científico no direcionamento de verbas de pesquisa a pesquisadores.

$\mathrm{Na}$ última década, a popularização do FI distorceu as decisões editoriais. Publicaram-se maus manuscritos, porém passíveis de serem citados, com erros considerados infames em periódicos com alto FI, em detrimento de manuscritos de alto valor científico. Por isto é que, segundo a opinião de vários autores, o FI tem uma influência negativa preocupante não só para os periódicos científicos como também para o desenvolvimento da ciência.

O FI e a sua forma de contagem e de determinação, segundo vários autores, é mal construído e mal utilizado como medida de qualidade científica. Abaixo citamos alguns pontos comumente aventados para justificar a assertiva acima, que consideram o índice como inapropriado: ${ }^{10,14-16}$

- A qualidade do material publicado não pode ser aferida através do tempo. A quantificação das citações, pelo período de dois anos, é arbitrária e foi determinada pelo ISI. Os artigos que compõem o denominador do cálculo do FI também são determinados pelo ISI e a regra de escolha destes artigos também não é clara e é duvidosa.

- O número dos periódicos incluídos na base de dados é mínimo em relação ao que, na realidade, é devidamente publicado.

- Os artigos de revisão são geralmente os mais citados quando comparados com os artigos originais, sendo que este dado favorece os periódicos que optam pela publicação deste tipo de contribuição como parte de estratégia editorial.

- O FI não discrimina as citações dos próprios autores de seus artigos, sendo que estes representam, na realidade, cerca de $1 / 3$ de todas as citações que são contabilizadas.

- O número de erros nas listas de referências é habitual e isto ocorre em aproximadamente $1 / 4$ de todas as referências citadas nos artigos, o que prejudica inevitavelmente a acurácia do FI.
- Assumir uma conectividade positiva entre as citações e o artigo produzido é equivocado, e muitas vezes o artigo cita artigos suspeitos ou de qualidade duvidosa. A citação não é considerada garantia de qualidade nem do artigo nem do citado.

Existe crítica exacerbada em relação ao FI sobre o fato de que o mesmo não deve ser utilizado como ferramenta de avaliação de pesquisa ${ }^{16,17}$ por ser determinado por tecnicismo, não se relacionando com a qualidade científica ou o conteúdo dos artigos. Alem dos óbices já citados anteriormente, o FI depende da área de abrangência e publicação da revista. As perspectivas de periódicos de áreas básicas são maiores quando comparadas com outros e que não estejam voltadas para temas de sucesso ou de evidência em um determinado momento, ${ }^{16}$ como ocorre atualmente com a área de terapia celular ou de células-tronco.

Em que pese o citado, as políticas editoriais adotadas têm tido influência na evolução do FI das revistas científicas. ${ }^{18}$ Em uma análise de sete periódicos médicos gerais (Arch Intern Med, BMJ, CMAJ, JAMA, Lancet, Med J Aust, $N$ Engl $J$ Med) em um período de 12 anos, ${ }^{18}$ revelou que os editores participaram ativamente, recrutando artigos com alto impacto e oferecendo melhoria e facilidades a estes autores. Ao observar, por exemplo, o Swiss Medical Weekly (SMW), é comum encontrar em suas páginas a oferta de suporte de estatística e outras facilidades para atrair contribuições, além do que alardeia que o seu FI ultrapassou a 1,5 e está aumentando. Assim estimula o envio de contribuições.

Esta política é contestada e determinadas abordagens ultrapassam o limite da ética na qual o objetivo claro é o de manipular o FI. ${ }^{19}$ Tais fatos são relatados no SMW, onde se contesta a manipulação do FI é se questiona se isto é uma obrigação do editor e cita alguns desvios éticos que passaremos a relatar.

No primeiro caso, um hematologista acusou um editor de uma revista de hematologia a obrigá-lo a inflar o número de referências com artigos já publicados na revista. Em outro caso, editores associados de uma revista foram denunciados por obrigarem um autor a citar artigos irrelevantes, no artigo submetido, oriundos de outras revistas. Autores receberam solicitações de editores para citarem o artigo submetido em outras revistas. São citadas também ressubmissões e adições de referências como carta ao editor. ${ }^{19}$

$\mathrm{Na}$ esteira desta discussão, várias outras propostas de substituição ou de modificação do uso do FI têm sido aventadas, e uma delas é do coeficiente de peso, o Weight Impact factor. ${ }^{20}$ Este índice não difere muito do FI tradicional, mas valoriza os artigos publicados em revistas de maior peso, tem peso $>$ de 1 e avalia os artigos em um período de três anos, diferenciando e valorizando os artigos originais. Outro índice proposto é o índice de prestígio ou Prestige factor (Pf), que segue na mesma linha. ${ }^{10}$

Apesar de todas as críticas citadas em 2006, o JCR analisou 6.088 periódicos, com um aumento de $32 \%$ no perío- 
do de 10 anos, ${ }^{21}$ e existe recente incremento e maior flexibilidade em aceitar novos periódicos, principalmente da América Latina, até então pouco representados no ISI. ${ }^{22}$ Objetivamente, o FI é considerado uma ferramenta imperfeita para mensurar do ponto de vista qualitativo, e o seu uso, para avaliação dos pesquisadores, não é inerente de riscos. ${ }^{21,23} \mathrm{~A}$ associação de ferramentas bibliométricas para esta finalidade é fundamental ${ }^{24,25,26}$ e estudos têm demonstrado que esta associação deve existir, principalmente se associando novas metodologias em decorrência do aumento de revistas eletrônicas e do acesso aos artigos serem cada vez mais frequentes através deste meio. ${ }^{27,28}$

\section{Conclusão}

Dentre as muitas opiniões conflitantes sobre o FI, ${ }^{29,30}$ existe um consenso de que o mesmo não é considerado uma ferramenta perfeita para medir a qualidade de um artigo, ou mesmo da produção científica dos pesquisadores e docentes, porém, isoladamente, não existe nada melhor ${ }^{30} \mathrm{e}$, por isto, ainda hoje, o índice deve ser considerado como um bom recurso técnico de avaliação científica.

\section{Abstracts}

In this report, the authors present information on bibliometric instruments and their importance in measuring the quality of scientific journals and researchers. They present the history and deployment of the impact factor by the Institute for Scientific Information that exists since 1955. Criticism regarding the inadequacy of the impact factor in the evaluation of scientific publications, its misuse and editorial handling strategies of this bibliometric index are presented and discussed. The new Capes classification for journals, based on various criteria and in particular the impact factor, is presented as is its influence on national scientific and academic life. The authors conclude that, despite all obstacles and discussions, the impact factor of the Institute for Scientific Information is still a useful tool and is the only one available to assess, in isolation, scientific and intellectual productivity. Rev. Bras. Hematol. Hemoter.

Key words: Impact factor; citation index; bibliometrics tools.

\section{Referências Bibliográficas}

1. Rocha e Silva M. In the February, 2008 issue of clinics. Clinics. 2008;63(1):1-2.

2. Procianoy RS. The journal impact factor today. J Pediatr (Rio J). 2007;83(6):487

3. Tague-Sutcliffe J. An introdution to informetrics. Information processing \& management. 1992;28(1):1-3.

4. Garfield E. Journal Impact factor: a brief review. CMAJ. 1999;161 (8):979-80.

5. Smith DR. Historical development of the journal impact factor and its relevance for occupational health. Ind Health. 2007; 45 (6):730-42.
6. Gross PL, Gross EM. College libraries and chemical education Science. 1927;66(1713):385-389, in Smith DR. Historical development of the journal impact factor and its relevance for occupational health. Ind Health. 2007;45(6):730-42.

7. Bush V. As we may think. Atlantic montly 1945;176:101-108 in Smith DR. Historical development of the journal impact factor and its relevance for occupational health. Ind Health. 2007; 45 (6):730-42.

8. Garfield E. Citation indexes for science; a new dimension in documentation through association of ideas. Science. 1955; 122 (3159):108-11.

9. Garfield E. The history and meaning of the journal impact factor. JAMA. 2006,295(1):90-3.

10. Walter G, Bloch S, Hunt G, Fisher K. Counting on citations: a flawed way to measure quality. Med J Aust. 2003;178(6):280-1.

11. Brown H. How impact factors changed medical publishing - and science. BMJ. 2007;334(7593):561-4.

12. López-Abente G, Muñoz-Tinoco C. Time trends in the impact factor of Public Health journals. BMC Public Health. 2005; $5: 24$.

13. Brasil. Ministério da Educação-MEC. Coordenação de Aperfeiçoamento de Pessoal de Nível Superior - Capes, Diretoria de Avaliação, Reestruturação do Qualis 2008. www.capes.gov.br

14. Greenwood DC. Reliability of journal impact factor rankings. BMC Med Res Methodol. 2007;7:48.

15. Colquhoun D. Challenging the tyranny of impact factors. Nature. 2003;423(6939):479.

16. Seglen PO. Why the impact factor of journals should not be used for evaluating research. BMJ. 1997;314(7079):498-502.

17. Ha TC, Tan SB, Soo KC. The journal impact factor: too much of an impact? Ann Acad Med Singapore. 2006;35(12):911-6.

18. Chew M, Villanueva EV, Van Der Weyden MB. Life and times of the impact factor: retrospective analysis of trends for seven medical journals (1994-2005) and their Editors' views. J R Soc Med. 2007; 100(3):142-50.

19. Sevinc A. Manipulating impact factor: an unethical issue or an Editor's choice? Swiss Med Wkly. 2004;134(27-28):410.

20. Habibzadeh F, Yadollahie M. Journal weighted impact factor: A proposal. Journal of Informetrics. 2008;2:164-172.

21. Andersen J, Belmont J, Cho CT. Journal impact factor in the era of expanding literature. J Microbiol. J Microbiol Immunol Infect. 2006;39(6):436-43.

22. Téllez-Zenteno JF, Morales-Buenrostro LE, Estañol B. Análisis del factor de impacto de las revistas científicas latinoamericanas Rev Med Chil. 2007;135(4):480-7.

23. Saha S, Saint S, Christakis DA. Impact factor: a valid measure of journal quality? J Med Libr Assoc. 2003;91(1):42-6.

24. Strehl L. O fator de impacto do ISI e a avaliação da produção científica aspectos conceituais e metodológicos. Ciência da informação. 2005;34(1).

25. Coura JR, Willcox LCB. Fator de impacto, produção científica e qualidade das revistas médicas brasileiras. Memórias do Instituto Oswaldo Cruz. 2003;98(3):293-7.

26. Mugnaini R. Caminhos para adequação da avaliação da produção científica brasileira: Impacto nacional versus internacional. 2006, Tese de doutorado apresentada ao Programa de Pós-graduação em Ciência da Informação, Escola de Comunicações e Artes da Universidade de São Paulo, São Paulo-253 p.

27. Piwowar HA, Day RS, Fridsma DB. Sharing detailed research data is associated with increased citation rate. PLoS One. 2007; 2(3): e308. 
28. Harnard S, Carr L. Earlier Web usage statistics as predictors of 28. later citation impact. Journal of the american society for information science 2006; 57(8): 1060-1072

29. Frank M., Impact factor: arbiter of excellence ? J Med Libr Assoc 2003; 91(1): 4-6
Avaliação: Editor e dois revisores externos

Conflito de interesse: sem conflito de interesse

Recebido: $25 / 08 / 2009$

Aceito: $31 / 08 / 2009$

\section{APÊNDICE 1}

Impact Factor confirms Nature is top research journal

Sexta-feira, 17 de Julho de 2009, 12:14.

De:

Este remetente é verificado pelo DomainKeys "Nature Publishing Group"

Adicionar remetente à lista de contatos

Para:milruiz@yahoo.com.br

Impact Factor confirms Nature is top research journal

Journal is highest-ranked multidisciplinary science title once again

Nature maintains its position as the number one weekly science journal, with a new Impact Factor of $31.434 *$.

Up from 28.751 last year, the new Impact Factor places Nature ahead of Science (28.103) and Cell (31.253), according to the 2008 Journal Citation Report (JCR). Nature also has the highest Eigenfactor; an alternative measure of journal impact, of all 6598 journals included the 2008 JCR.

The impact factor is a measure of the frequency with which the average article in a journal has been cited in a particular year. The impact factor helps to evaluate a journals relative importance, especially when compared with others in the same field.

In a separate indicator of excellence, Nature was last week named journal of the century' by the BioMedical \& Life Sciences Division (DBIO) of the Special Libraries Association (SLA). Voted for by DBIOs 686 members, the award was given to celebrate SLA's Centennial and recognizes the most influential journal of the last 100 years (1909-2009).

To celebrate we are offering you an exclusive $30 \%$ discount if you subscribe to Nature this week.**

http://links.ealert.nature.com/http://links.ealert. nature.com/ctt?kn=19\&m=33636304\&r=MTc3MTE5NDg5NwS2\&b $=2 \& j$ $=\mathrm{NTMzOTU} 5 \mathrm{MzQS} 1 \& \mathrm{mt}=1 \& \mathrm{rt}=0$

*2008 Journal Citation Report (Thomson Reuters, 2009)

**applies to personal subscriptions only, taken before 1st July 2009

As a registered user of Nature Publishing Groups Web sites, our database indicates that you have opted-in to receive NPG Product Information and Special Offers. If you no longer wish to receive these emails or to discontinue all email services from Nature Publishing Group, please update your online account. www.nature.com/nams/svc/myaccount

(You will need to log in to be recognised as a Nature registrant)

For further technical assistance, please contact our registration department mail to: registration@nature.com

For print subscription enquiries, please contact our subscription department mailto:subscriptions@nature.com

For other enquiries, please contact our customer feedback department mailto:feedback@nature.com

Nature Publishing Group

75 Varick Street, 9th floor | New York | NY 10013-1917 USA

Nature Publishing Groups worldwide offices:

London, Paris, Munich, New Delhi, Tokyo, Melbourne, San Diego, San Francisco, Washington, New York, Boston, Hong Kong, Gurgaon, Mexico City, Basingstoke.

Macmillan Publishers Limited is a company incorporated in England and Wales under company number 785998 and whose registered office is located at Brunel Road, Houndmills, Basingstoke, Hampshire RG21 6XS.

(C) 2009 Nature Publishing Group, a division of Macmillan Publishers Limited.

All rights reserved 